concerning hippocampal injury with febrile seizures.

Interictal EEG spikes for seizure lateralization in mesial temporal lobe epilepsy. In a study of 21 patients at the Universitatsklinik fur Neurologie, Vienna, Austria, lateralization of clinical seizures was correct (ipsilateral to side of hippocampal sclerosis) in almost $100 \%$ of cases with unitemporal spikes and in only $50 \%$ of those showing bitemporal spikes (Serles W et al. Clinical seizure lateralization in mesial temporal lobe epilepsy. Differences between patients with unitemporal and bitemporal interictal spikes. Neurology March 1998;50:742-747).

\title{
TEMPORAL LOBE MALFORMATIONS AND EPILEPSY
}

Temporal lobe developmental malformations (TLDM) (focal cortical dysplasia and balloon cells) occurred with mesial temporal sclerosis as dual pathologies in $87 \%$ of 30 patients with unilateral TLDM and intractable partial epilepsy treated at the UAB Epilepsy Center, University of Alabama at Birmingham, AL. A quantitative MRI analysis with the inclusion of a normalization process was used for the detection of bilateral hippocampal formation atrophy when visual analysis, using optimal protocol including IR and FLAIR sequences, failed. The dual pathologies might be developmental or the hippocampi could be damaged secondarily by a kindling effect of repeated seizures from the TLDM. The surgical implications of the dual pathology are discussed. (Ho SS, Kuzniecky RI, Gilliam F, Faught E, Morawetz R. Temporal lobe developmental malformations and epilepsy. Dual pathology and bilateral hippocampal abnormalities. Neurology March 1998;50:748-754). (Reprints: Dr Ruben I Kuzniecky, Department of Neurology, UAB Station, Birmingham, AL 35294).

COMMENT. Mesial temporal sclerosis can occur in association with temporal lobe focal dysplasia in patients with refractory temporal lobe epilepsy. These authors have previously demonstrated that the MRI will identify lesions with moderate to severe histologic abnormalities, but may not detect mild neuronal and cortical dysplasias. Dual pathology in patients with mesial sclerosis may be underdiagnosed by MRI and may explain the occurrence of temporal lobe epilepsy as a sequel to complex febrile seizures in some cases.

Of 67 patients with medial temporal lobe seizures controlled by temporal lobectomy at Yale University and Epilepsy Center, 45 (67\%) had histories of febrile seizures before 5 years of age, and of these, 33 had complex febrile seizures lasting longer than 30 minutes. The duration of the febrile convulsion was the most important predictor of temporal lobe epilepsy (TLE) in a study at the University of Western Ontario, London, Ontario. The mean duration of the FC was $100 \mathrm{~min}$ in patients with TLE, and $9 \mathrm{~min}$ in those without TLE. (See Progress in Pediatric Neurology III, PNB Publishers, 1997;pp19 and 32)).

\section{DUAL ETIOLOGY OF RASMUSSEN'S SYNDROME}

Five patients with Rasmussen's syndrome reported from the Montreal Neurological Institute had the typical findings of chronic encephalitis together with tuberous sclerosis, tumor, or vascular abnormality discovered on pathological examination of tissue removed at operation. Dual pathologies were found in $10 \%$ of the patients in the authors' series. (Hart YM, Andermann F, Robitaille Y et al. Double pathology in Rasmussen's syndrome. A window on the etiology? Neurology March 1998;50:731-735). (Reprints: Dr F Andermann, Montreal Neurological Institute, 3801 University Street, Montreal, Quebec H3A 2B4). 